\title{
Physicochemical Studies, Characterization \& Adsorption Capacity of Activated Carbon Derived from Dathura Stronsium Fruit Shell
}

\author{
Kavita Gour
}

\begin{abstract}
In the Present investigation various activated carbon derived from waste material of plant has been synthesized .These carbon materials have high porosity.This high degree of porosity of adsorbents is associated with large surface area, which makes it an excellent adsorbent for removal of Heavy Metals. The activated carbon has advantages over the other with respect of its adsorption capacity, operating Characteristic \&Cost. The activated carbon materials have been characterized by surface area, particle size, bulk density, Volatile matter, Moisture content, Ash content, FTIR \&SEM studies. The goal of the present investigation was an attempt to develop low cost, highly effective and simple to use activated carbon materials for removal $\mathrm{Cr}(\mathrm{VI})$, Cd(II) and Zn(II) from contaminated water based on adsorption phenomenon. This newly developed adsorbent has been proved to be more promising for heavy metals removal without affecting the quality of treated water.
\end{abstract} Metals

Keywords: Adsorbent, Activated Carbon, Porosity, Heavy

\section{INTRODUCTION}

There is fair number of manufactures of granular as well as powdered activated carbon materials. The adsorption capacity of activated carbon depends not only upon the textural Properties but also the chemical nature of its surface. The surface of activated carbon may contain number of oxygen containing functional groups. The nature and surface properties of any activated carbon can be changed by different treatments. The property of activated carbon to remove specific contaminants depends on the presence of certain functional groups on the surface of adsorbing material, which strongly influences the adsorption process. However many of these carbon materials may not be necessarily suited for wastewater treatment system. In addition, some activated carbon has an advantage over the other with respect to its adsorption capacity depending on the adsorb ate to be adsorbed, physical durability, operating characteristics and cost. In this way adsorbents under consideration in the present research work are suitable for the treatment of water and wastewater with special reference to abatement of toxic heavy

Manuscript received on 25 April 2021 | Revised Manuscript received on 8 May 2021 | Manuscript Accepted on 15 May 2021| Manuscript published on 30 May 2021.

* Correspondence Author

Dr. Kavita Gour*, Associate Professor, Department of Chemistry, Priyadarshini J L College of Engineering Nagpur, India. Email: kavitapce@gmail.com

(C) The Authors. Published by Lattice Science Publication (LSP). This is an open access article under the CC-BY-NC-ND license (http://creativecommons.org/licenses/by-nc-nd/4.0/)

\section{SYNTHESIS OF ADSORBENT}

Activated charcoal is a form a carbon within increased surface area for adsorption. It is also referred is "activated carbon, active charcoal or at times activated coal.

Activated charcoal is a form a carbon within increased surface area for adsorption. It is also referred is "activated carbon, active charcoal or at times activated coal." In order to produce activated carbon, a carbon source is treated under certain condition to increase its surface area or number of pores.The fruit shells of Datura stramonium were cut into small pieces and washed several times with water followed by distilled water and dried in sunlight to remove the moisture. The carbonization of the fruit shells was performed in a muffle furnace.The chars were subjected to thermal activation in the absence of air at elevated temperature $900^{\circ} \mathrm{C}$ and held at that temperature for $1 \frac{1 / 2}{2}$ hour. The carbonaceous adsorbents were obtained viz. ACDSF. The adsorbent were grind and sieved through 200 mesh sieves.

\section{A Steps Involved For Activation Process}

The activation process of carbon is carried out by treated with $25 \%$ concentrated solution of zinc chloride[1].The pre weighted dried ACDSC were transferred into a clean different plastic pail. 25\% zinc chloride solution was poured into the plastic pail. The amount of zinc chloride solution was sufficient to soak ACDSC completely into the solution. The plastic pail was covered with lid and kept for 20-24 hours. pail. The material was washed and then rinsed thoroughly with double distilled water to removes excess of zinc chloride. They was washed with hot hydrochloric acid (1:1) for the removal of traces of zinc chloride and the mineral ingredients. After acid washing, the materials were again washed with hot double distilled water until the acidity and the chloride content was completely removed. The product was dried in hot air over at $110^{\circ} \mathrm{C}$ for about 24 hours. They was further sieved through 100 mesh sieve and stored in stoppered plastic bottles.

\section{B. Characterization of the Adsorbents}

The activated carbon materials have been characterized by surface area, particle size, bulk density, Volatile matter, Moisture content, Ash content, FTIR \&SEM studies 
Table -I Characteristics of the Adsorbents

\begin{tabular}{|c|c|}
\hline & ACDSFS \\
\hline Properties & \\
\hline Particle size (mm) & 0.81 \\
\hline Bulk density (gm/cc) & 0.332 \\
\hline Moisture content (\%) & 1.59 \\
\hline Volatile Matter (\%) & $11.50 \%$ \\
\hline Ash Content (\%) & $6.30 \%$ \\
\hline Water Soluble Matter & $0.66 \%$ \\
\hline Acid Soluble Matter & $1.86 \%$ \\
\hline Surface Area (m²/g) & 291 \\
\hline
\end{tabular}

\section{RESULT \& DISCUSSION}

The particle size has great impact on the adsorption efficiency of adsorbent when they were applied as an adsorbent in water and wastewater treatment. The newly prepared activated carbon materials i.e. ACDSFS, was sieved to achieve particular particle size. The particle size of all the newly synthesized adsorbents was in the range of $0.81 \mathrm{~mm}$. which is indicative of good quality of adsorbent and prove its ability for rapid adsorption.

Bulk density may be regarded as the measure of activity of adsorbents. Higher is the bulk density, faster the settling rate and lower the volume of sludge to be handled and dewatered after Treatment. Bulk density varies with the alteration of raw materials used and the process of activation. It also roughly gives an idea regarding the degree of activation and inversely proportional to the surface area 2] The bulk densities of the adsorbents under study was found to be reasonably good i.e. in the range of $0.332 \mathrm{gm} / \mathrm{cc}$.

Moisture content does not affect the adsorptive power of the adsorbent but dilutes the adsorbent and necessitates the use of additional weight of adsorbent to provide the required weight (dosage). Moisture content noticed in all the adsorbents under study is found considerably very low i.e. in the range of $1.59 \%$.

Volatile matters generally found in the adsorbent include methane, hydrocarbons, hydrogen and carbon monoxide and incombustible gases like carbon dioxide, nitrogen etc. Thus, volatile matter is an index of all the gaseous fuels present. Volatile matter is found in adsorbent in the range of $11.50 \%$. The experimental result proved that newly synthesized adsorbents i.e. ACDSFS, contains considerably low value of volatile matter

The ash content, generally gives an ideal about inorganic constituents associated with the adsorbent. ACDSFS contains slightly higher ash content i.e. 6.30\% .From obtained data the acid soluble matter is slightly higher than water-soluble matter. Acid soluble matter is high in A Surface area is one of the most important criteria in deciding the adsorption capacity of adsorbents. Generally, larger the surface area of activated carbon, higher the adsorption capacity. In the present investigation, the surface area was determined by the standard methylene blue method[3] Surface area varies with surface of raw materials and activation process. The surface area of self prepared activated carbon material was found to be in the range of $291 \mathrm{~m} 2 / \mathrm{gm}$.

The surface area provide the effectiveness of the adsorbing material for a particular use[4] But in a real sense, the adsorptive capacity of any activated carbon material depends not only the available surface area but also on the quality and quantity of the functional group present on the surface and also on the surface morphology of the activated carbon materials.

\section{A. FTIR Studies of ACDSFS}

FTIR spectrum of ACDSFS has been shown in Fig. 1. Appearance of a band at $3000-3700 \mathrm{~cm}-1$ corresponds to stretching vibration of phenolic hydroxyl $(-\mathrm{OH})$ group and $\mathrm{NH}$-stretching vibration of nitrogen atom of amino groups. The band for $-\mathrm{OH}$ stretching in the range below $3700 \mathrm{~cm}-1$ was assigned by Zawadzki[3] A peak at $2310 \mathrm{~cm}-1$ shows more strongly hydrogen bonded $-\mathrm{OH}$ group. The band observed at $2850 \mathrm{~cm}-1$ corresponded to $\mathrm{C}-\mathrm{H}$ stretching vibrations. A sharp peak at appearing at $1597 \mathrm{~cm}-1$ may be due to $\mathrm{C}=\mathrm{N}$ stretching vibration. The

peaks appeared at 1440 and $1167 \mathrm{~cm}-1$ are due to methylene bridges coupled with aromatic ring[4] A peak at $754.54 \mathrm{~cm}-1$ band showed $\mathrm{CH} 2$ rocking. Absorption band at $1277 \mathrm{~cm}-1$ could be attributed to $\mathrm{C}-\mathrm{OH}$ stretching. The peak at $1152.82 \mathrm{~cm}-1$ and $1078.19 \mathrm{~cm}-1$ corresponding to $\mathrm{C}-\mathrm{O}-\mathrm{C}$ asymmetric stretching vibration. The additional peak at $661.82 \mathrm{~cm}^{-1}$ can be assigned to bonding modes of aromatic compound

\section{B. SEM Studies of ACDSFS}

SEM micrograph of ACDSFS is shown in Fig.2. SEM image of activated carbon has been obtained examined using an accelerating voltage of $15 \mathrm{kv}$ at x2500 magnification. Image shows that the surface of ACDSF is well developed and closely packed accompanied with many holes and cavities. The surface also accompanied with many of thin sheets or layers. Such micrograph is indicative of the adsorbent which would have created more surface area available for better adsorption.

\section{Adsorption Studies on Activated Carbon derived from Datura Stramonium Fruit Shell (ACDSFS)}

The adsorption capacity of adsorbent was determined. The effect of different experimental conditions such as contacttime, pH\&adsorbent dose on adsorption capacity was studied

\section{Effect of Contact Time on Adsorption}

Effect of contact time between adsorbent and adsorbate is one of the important parameter in the studies of adsorption process. 
The effect of contact time on percent removal of metal ions has been shown in Fig.3 The dependence of heavy metal ion adsorption was studied by

varying the contact time of ACDSFS, while other parameters such as $\mathrm{pH}$, \&Adsorbent dose were kept optimum.

The percentage removal of $\mathrm{Cd}(\mathrm{II}), \mathrm{Cr}(\mathrm{VI}) \& \mathrm{Zn}(\mathrm{II})$ is $88.37,85.17 \% \& 98.37 \%$ respectively at contact time $120,100 \& 110 .(6)$

\section{E. Effect of pH on Adsorption}

The role of hydrogen ion concentration on adsorption process was examined in aqueous solutions as function of $\mathrm{pH}$. $\mathrm{pH}$ influences the surface charge of the adsorbents. So the $\mathrm{pH}$ of the aqueous solution is an important controlling parameter in the heavy metal adsorption process.

The dependence of heavy metal ion adsorption was studied by varying the $\mathrm{pH}$ of ACDSFS ,while other parameters such as contact time, \&Adsorbent dose were kept optimum. The effect of $\mathrm{pH}$ on percent removal of metal ions has been shown in Fig.4

The percentage removal of $\mathrm{Cd}(\mathrm{II}), \mathrm{Cr}(\mathrm{VI}) \& \mathrm{Zn}(\mathrm{II})$ is $89 \%, 89.34 \% \& 95.24$ respectively at $\mathrm{pH} 6 \& 4(6)$

\section{F. Effect of Adsorbent Dosages}

The dependence of heavy metal ion adsorption was studied by varying the amount of ACDSFS adsorbent from 1 to $10 \mathrm{gm} / \mathrm{lit}$, while other parameters such as $\mathrm{pH}$, contact time and concentration of adsorbate were kept optimum. The percentage removal of $\mathrm{Cd}(\mathrm{II}), \mathrm{Cr}(\mathrm{VI}) \& \mathrm{Zn}(\mathrm{II})$ is $90.6 \%, 84.34 \% \& 97.6$ respectively at adsorbent dose $6,5, \& 6$ mg per litre(6)

\section{CONCLUSION}

The various physicochemical results\& adsorption capacity of ACDSFS indicates that activated carbon derived from Datrastramonium fruit shell a low cost adsorbent. It is useful for the removal of Heavy Metals Cd(II),Cr(II)\&Zn(II) from aqueous solution. From the result it is clear that all operational parameter such as contact time, $\mathrm{pH}$, and Adsorbent dose affect the removal efficiency of Heavy metals. Thus the activated carbon derived from Daturastronium fruit shell has been eco friendly \&low cost adsorbent material.

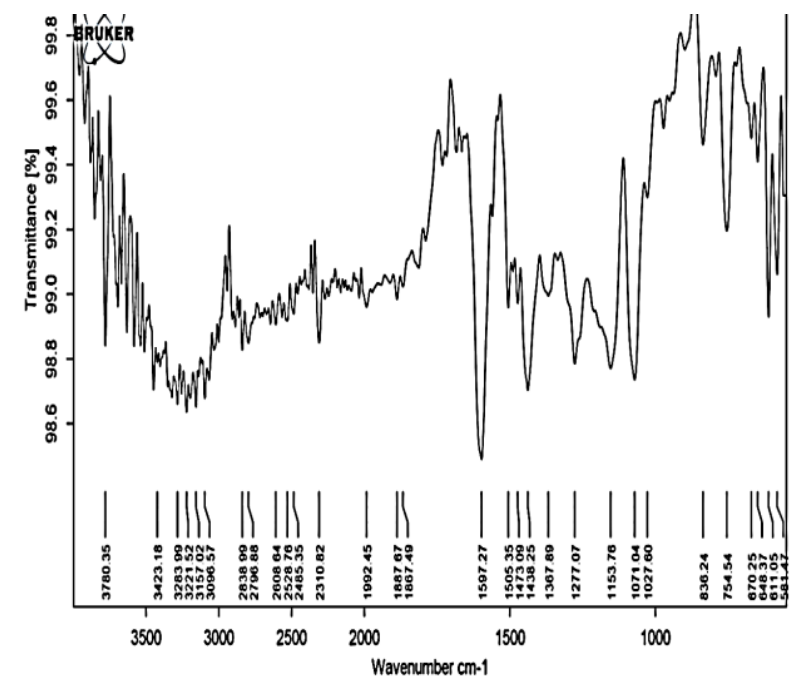

Fig. 1. FTIR of Adsorbent

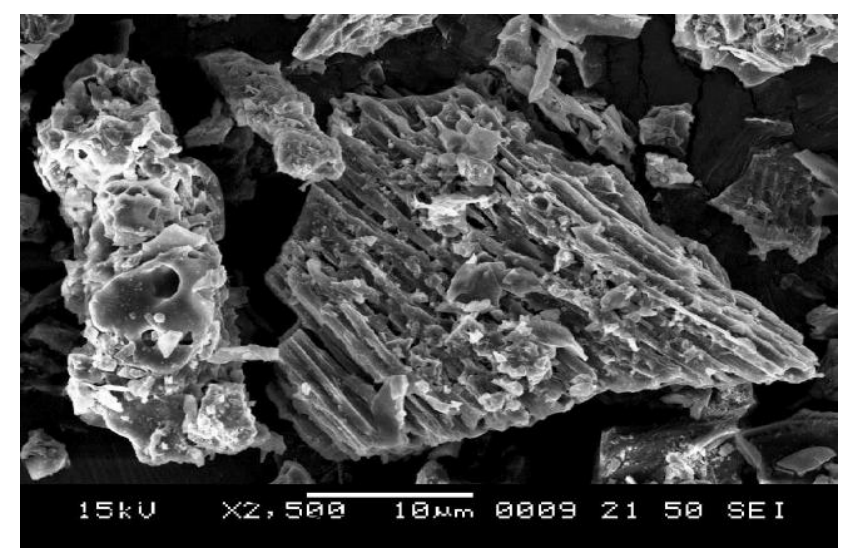

Fig .2 . SEM of Adsorbent

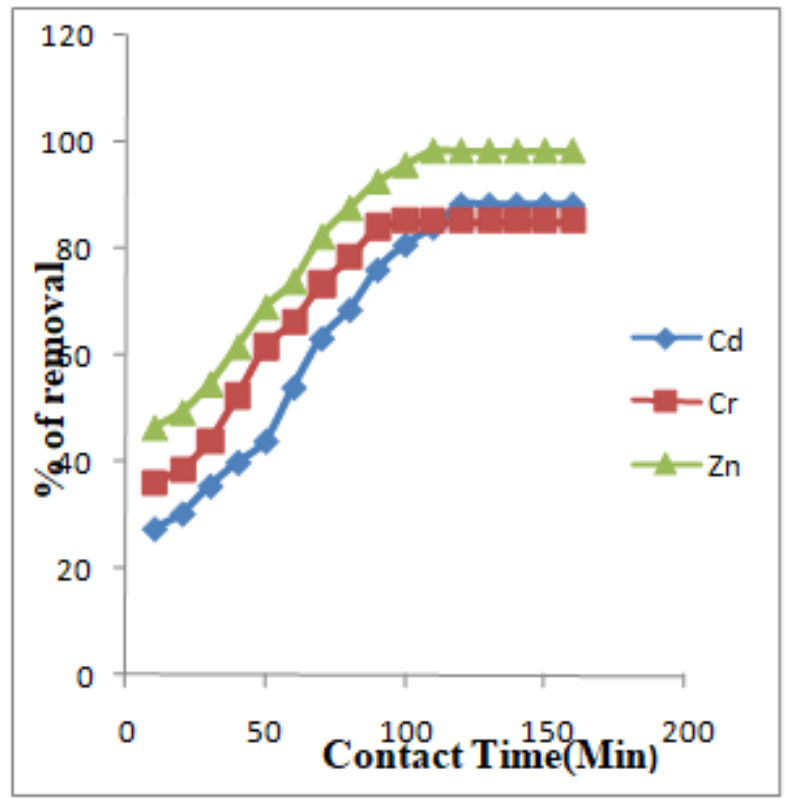

Fig.3. Effect of Contact Time on Cd(II), Cr(VI), and $\mathrm{Zn}(\mathrm{II})$ removal by ACDSFS

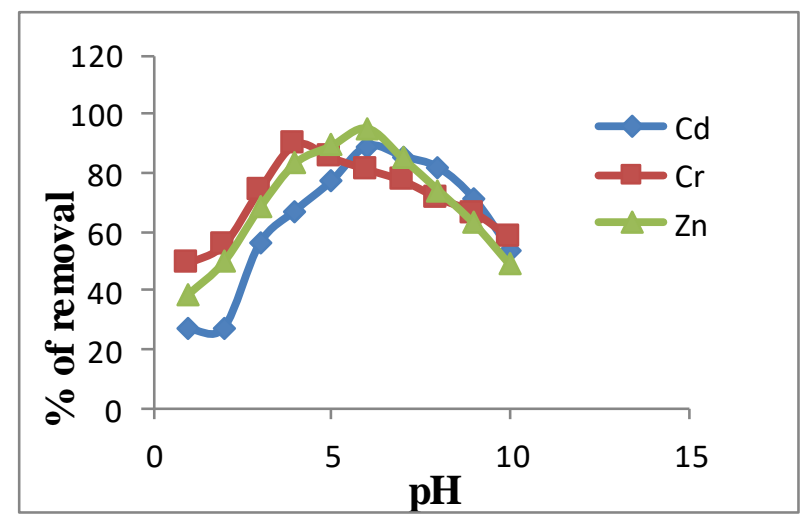

Fig.4. Effect of pH on Cd(II), Cr(VI) and Zn(II) removal by ACDSFS

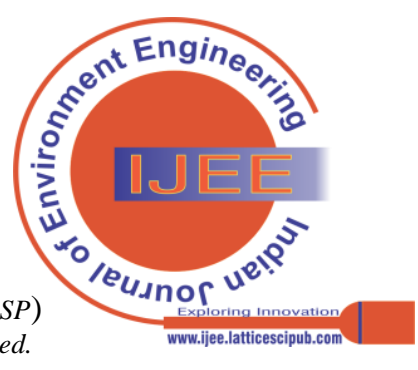




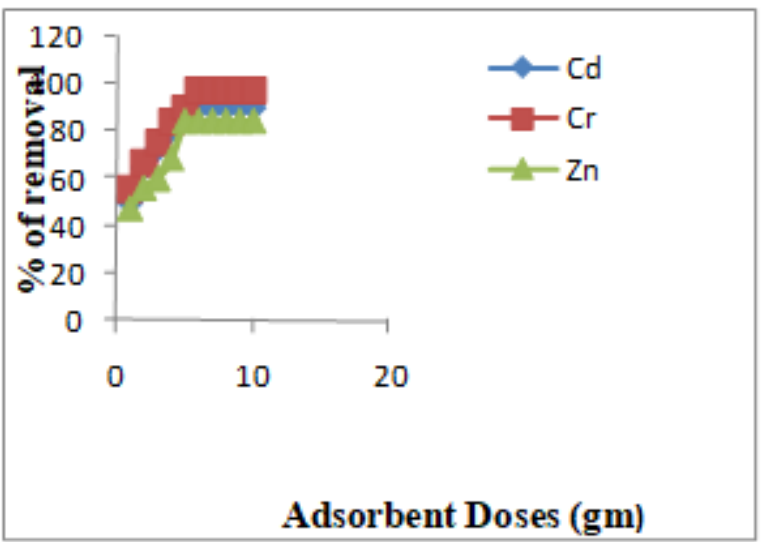

Fig.5. Effect of Adsorbent Doses on Cd(II), Cr(VI) and $\mathrm{Zn}(\mathrm{II})$ removal

\section{REFERENCES}

1. Giles c.h., trivedi a.s., "specific surface area by methylene blue method," chem. Ind. 40; 1426, (1969).

2. Weber w.j., "sorption from solution by porous carbon," principle \& application of water chemistry edn. (1965).

3. Mccreamy j.j. \& snoepnik y.l., " granular activated carbon in water treatment," j.awwa; vol.68(8); 437-444,(1977). [CrossRef]

4. Zawazki j; ir spectroscopy of oxygen surface compounds on carbon, carbon, 16, 491-497, (1978). [CrossRef]

5. Gurnule w.b, rahangdale p.k., paliwal 1.j. And kharat r.b., "synthesis, characterization and ion-exchange properties of 4-hydroxyacetophenone, biuret and formaldehyde terpolymer resin." Reactive \& functional polymer, 55, 255-265 (2003). [CrossRef]

6. Gour k \&donadkar d"adsorptive removal of toxic cd(ii)from waste water using activated carbon prepared fromdatura stramonium fruit shell”aip conference proc 2104,030042, ,012013,(2019) [CrossRef]

\section{AUTHORS PROFILE}

Dr. Kavita Gour is working as Dean \& Associate Professor in Chemistry at Priyadarshini J L College of Engineering,Nagpur Having an experience of 26 yers of teaching \& 3 years of research (DAE project)Mumbal \&Guided many students for PhD \& PG projects. She has 70 research pepers in her credit. Which are published in reputed Journal. he
authored two books. Having life membership of various profession body.

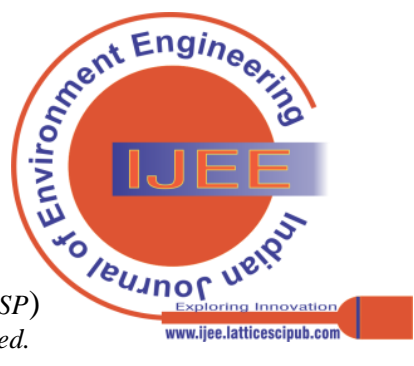

